\title{
Gravidez e Hemodiálise: A Propósito de uma Gravidez Gemelar Bem Sucedida
}

\section{Pregnancy and Hemodialysis: About a Successful Twin Pregnancy}

\author{
Rita MEDEIROS ${ }^{1}$, Maria de São José PAIS ${ }^{1}$, Luís FREITAS ${ }^{2}$, Paulo MOURA
}

Acta Med Port 2021 Jan;34(1):56-58 · https://doi.org/10.20344/amp.11377

\section{RESUMO}

A gravidez é uma situação rara em mulheres com doença renal crónica sob hemodiálise. Contudo, a sua incidência tem aumentado nos últimos anos. Apesar de ser uma situação de elevado risco materno e fetal, tem havido uma melhoria na assistência, devido ao aumento do número de casos descritos e da experiência adquirida na terapia dialítica. Relatamos o caso de uma multípara de 32 anos com hipertensão crónica e doença renal crónica com 10 anos de evolução e difícil controlo, com diagnóstico de gravidez gemelar bicoriónica/biamniótica, quatro meses após início da hemodiálise. A gestação foi vigiada por uma equipa multidisciplinar, as sessões de hemodiálise bem toleradas clínica e hemodinamicamente, com bom controlo tensional e analítico seriado, assim como adequado desenvolvimento de ambos os fetos. Foi submetida a cesariana às 34 semanas e seis dias por início de trabalho de parto, tendo nascido dois meninos com peso adequado para a idade gestacional.

Palavras-chave: Diálise Renal; Gravidez Gemelar; Insuficiência Renal Crónica

\section{ABSTRACT}

Pregnancy is a rare condition in women with chronic kidney disease on hemodialysis, but its incidence has increased in recent years. Despite being a high-risk condition for both mother and fetus, there has been an improvement in care due to the increased number of cases described and the experience gained in dialysis therapy. We report the case of a multiparous 32-years-old women with chronic hypertension and chronic kidney disease with 10 years of evolution and difficult control, with a diagnosis of dichorionic/diamniotic twin pregnancy, 4 months after beginning hemodialysis. Pregnancy was managed by a multidisciplinary team, hemodialysis sessions were clinically and hemodynamically well tolerated, with good blood pressure and analytical control, as well as adequate development of both fetuses. She went into labor at 34 weeks and 6 days, so a caesarean section was performed, with 2 boys born with adequate weight for gestational age.

Keywords: Pregnancy, Twin; Renal Dialysis; Renal Insufficiency, Chronic

\section{INTRODUÇÃO}

A gravidez em doentes com doença renal crónica (DRC) sob hemodiálise é uma situação rara, com uma incidência estimada de $1 \%$ - $7 \% .^{1-4}$ As alterações metabólicas e endócrinas condicionam diminuição dos ciclos ovulatórios e um ambiente intrauterino hostil. ${ }^{1,5-7} \mathrm{~A}$ amenorreia secundária atrasa o diagnóstico da gravidez e subsequentes cuidados pré-natais adequados.

A prematuridade e baixo peso são as complicações obstétricas mais frequentes. Todavia, o número de gravidezes bem sucedidas tem aumentado, com um ganho na sobrevivência fetal de aproximadamente $25 \%$ por década (superior a $90 \%$ nos últimos anos). 2,7,8 Atualmente considera-se que os principais fatores preditivos de agravamento da função renal residual e complicações obstétricas são o estádio da DRC e a existência de outras comorbilidades como hipertensão arterial (HTA). ${ }^{8-10} \mathrm{O}$ desafio é manter o ambiente intrauterino favorável ao desenvolvimento fetal, sendo fundamental uma compreensão das mudanças fisiológicas da gravidez e estreita colaboração multidisciplinar.

\section{CASO CLÍNICO}

Doente do sexo feminino, 32 anos, caucasiana, antecedentes obstétricos de três gestações (três cesarianas), a última complicada por pré-eclâmpsia sobreposta a hipertensão crónica, com posterior diagnóstico de DRC (glomerulosclerose segmentar focal em biópsia renal). Submetida a tratamento imunossupressor com ciclofosfamida e corticoterapia apresentando boa resposta inicial mas recidiva precoce. Abandonou a consulta, estando sem orientação terapêutica, entre 2011 e novembro de 2013, altura em que recorreu ao serviço de Urgência da área de residência por crise hipertensiva, com subsequente necessidade de iniciar diálise.

Em março de 2014, por queixas de distensão abdominal, realizou ecografia abdominal que revelou gravidez gemelar bicorionica/biamniótica de 19 semanas. A estratégia dialítica foi otimizada para quatro sessões de cinco horas por semana e referenciada para consulta de Obstetrícia/Nefrologia do nosso serviço. Na primeira avaliação, às 21 semanas, ambos os fetos apresentavam morfologia normal, biometrias no percentil 25 - 50 e 50 - 75, respetivamente, e índices de líquido amniótico (LA) normais. Manteve consultas com avaliação ecográfica a cada três semanas até às 27 semanas, altura em que foi decidido internamento para vigilância mais apertada e necessidade de reajuste do programa de hemodiálise para seis sessões

1. Departamento de Obstetrícia A. Maternidade Doutor Daniel de Matos. Centro Hospitalar e Universitário de Coimbra. Coimbra. Portugal.

2. Departamento de Nefrologia. Centro Hospitalar e Universitário de Coimbra. Coimbra. Portugal.

$\triangle$ Autor correspondente: Rita Medeiros. rita.adriana.medeiros@gmail.com

Recebido: 03 de outubro de 2018 - Aceite: 17 de junho de 2019 | Copyright $\odot$ Ordem dos Médicos 2021 
por semana, difícil de conciliar com a distância à área de residência. Este tratamento foi bem tolerado clínica e hemodinamicamente, mantendo controlo tensional sob nifedipina $30 \mathrm{mg}$ em toma diária e controlo analítico seriado estável. Na sequência dos exames de rotina do segundo trimestre (PTGO 61/156/175 mg/dL), foi feito o diagnóstico de diabetes gestacional, que foi controlada com insulinoterapia. Realizou corticoterapia para maturação pulmonar fetal às 28 semanas e iniciou terapêutica de suporte com eritropoietina ( $1 \mathrm{x} /$ semana), carbonato de cálcio, sulfato ferroso e enoxaparina. Ambos os fetos apresentaram crescimento no percentil 50, LA e fluxometrias das artérias umbilicais normais. Às 34 semanas e seis dias, por início do trabalho de parto, foi realizada cesariana. Nasceram dois meninos com $2220 \mathrm{~g}$ e $2260 \mathrm{~g}$ de peso e índice de Apgar 8/9/10 em ambos. No pós-parto manteve-se estável, com ajuste das sessões de hemodiálise para três vezes por semana.

\section{DISCUSSÃO}

A gravidez em mulheres com DRC apresenta baixa incidência, podendo ser efeito direto da doença na fertilidade ou representando uma subnotificação desta condição. ${ }^{9} \mathrm{~A}$ anemia e hiperprolactinémia, associadas frequentemente a amenorreia e anovulação, contribuem para a reduzida fertilidade. ${ }^{2,7}$ Quando a gravidez ocorre, o diagnóstico é frequentemente tardio uma vez que irregularidades menstruais, dores abdominais, náuseas e vómitos são sintomas frequentes nestas doentes. ${ }^{1,11}$

Em 1971 foi feita a primeira descrição de gravidez sob diálise por Confortini et al e, desde então, um crescente número de casos tem sido relatado com informações discordantes quanto ao prognóstico maternofetal.5,12,13 Embora seja uma condição de risco, os avanços e experiência dos últimos anos têm conduzido a um incremento do número de gravidezes sob diálise. ${ }^{11,12,14}$ A gravidez é bem sucedida na maioria das mulheres cuja função renal se encontra normal ou discretamente alterada. Contudo, perante algum grau de insuficiência renal prévia à conceção, existe um aumento significativo de complicações maternas e/ou perinatais. ${ }^{1,9,13}$ É fundamental uma vigilância multidisciplinar (Obstetra, Nefrologista e Neonatologista), com especial ênfase na prevenção e tratamento das eventuais complicações.

Apesar da melhoria dos resultados perinatais, aproximadamente metade das gestações apresentam alguma complicação. ${ }^{13}$ As principias complicações maternas são as doenças hipertensivas, podendo ser difícil o diagnóstico diferencial entre pré-eclâmpsia e descompensação da DRC. ${ }^{1,9}$ A HTA não controlada e pré-eclâmpsia são o principal fator de risco para a ocorrência de prematuridade e outros desfechos adversos (baixo peso, síndrome de dificuldade respiratória, necessidade de cuidados intensivos neonatais), pelo que deverá ser rigorosamente monitorizada e otimizada. ${ }^{3,8}$ Neste caso, a paciente apresentava hipertensão crónica e antecedentes de pré-eclâmpsia, tendo sido conseguido controlo tensional com fármacos orais associados à otimização da estratégia dialítica. A anemia é outra complicação frequente, a qual se deve não só às alterações fisiológicas da gravidez, como à deficiência de ferro e eritropoietina originada pela DRC e hemodiálise. A suplementação com ferro é recomendada para manter uma ferritina entre 100 - 500 ng/L. A eritropoietina tem-se mostrado segura na gravidez, sendo recomendada para manter a hemoglobina entre 9,5 - 11,5 g/dL..$^{1,3,11,15}$ A ocorrência de desequilíbrios eletrolíticos deve ser evitada e corrigida quando detetada. ${ }^{3,8,11}$

As principais complicações fetais incluem restrição de crescimento, hidrâmnios, parto pré-termo (PPT) e morte intrauterina ou neonatal. ${ }^{2,3,7-9,15} \mathrm{~A}$ taxa de prematuridade é alta e, consequentemente, a de recém-nascidos com baixo peso. Muitas mortes fetais e PPT não se devem a intervenções obstétricas para proteger a mãe, mas sim a complicações obstétricas. ${ }^{4}$ É recomendada vigilância ecográfica regular do crescimento fetal. A administração de corticoterapia antes e surfactante após o parto são recomendados para promover a maturação pulmonar fetal. Neste caso, o parto ocorreu às 34 semanas, ressalvando ser uma gravidez gemelar, já por si com risco acrescido de PPT, apresentando os recém-nascidos peso adequado, sem necessidade de suporte na unidade de cuidados intensivos.

A principal alteração no regime dialítico em grávidas é o aumento substancial do tempo de diálise, verificando-se uma taxa de nados vivos superior a $85 \%$ nos regimes de duração superior a $36 \mathrm{~h} /$ semana comparado com $48 \%$ nos regimes com duração inferior a $20 \mathrm{~h} /$ semana. ${ }^{3-5,11}$ Este caso corrobora a estabilidade hemodinâmica conseguida com o aumento das sessões como importante fator de sucesso.

\section{CONCLUSÃO}

A incidência de gravidez em mulheres sob diálise é baixa, não havendo estudos que permitam uma adequada avaliação da frequência de gestações ou dos resultados perinatais. Apesar dos dados controversos, tem havido uma melhoria na assistência, devido ao aumento do número de casos descritos e da experiência adquirida nomeadamente com a alteração dos regimes dialíticos. É fundamental iniciar a vigilância pré-natal o mais precocemente possível e por equipa multidisciplinar.

Este caso é peculiar uma vez que é raro encontrarmos na literatura descrições de gravidezes múltiplas com bom desfecho materno fetal, considerando todas as singularidades deste tipo de gestações.

\section{PROTECÇÃO DE PESSOAS E ANIMAIS}

Os autores declaram que os procedimentos seguidos estavam de acordo com os regulamentos estabelecidos pelos responsáveis da Comissão de Investigação Clínica e Ética e de acordo com a Declaração de Helsínquia da Associação Médica Mundial actualizada em 2013.

\section{CONFIDENCIALIDADE DOS DADOS}

Os autores declaram ter seguido os protocolos do seu centro de trabalho acerca da publicação de dados. 


\section{CONSENTIMENTO DO DOENTE}

Obtido.

\section{CONFLITOS DE INTERESSE}

Os autores declaram a inexistência de conflitos de interesse na realização do presente trabalho.

\section{REFERÊNCIAS}

1. Alkhunaizi A, Melamed N, Hladunewich MA. Pregnancy in advanced chronic kidney disease and end-stage renal disease. Curr Opin Nephrol Hypertens. 2015;24:252-9.

2. Chou CY, Ting IW, Lin TH, Lee CN. Pregnancy in patients on chronic dialysis: a single center experience and combined analysis of reported results. EJOG. 2008;136:165-70.

3. Mercadal L, Nizard J. Prepregnancy counselling and management of pregnancy in haemodialysis patients. Nephrol Dial Transplant. 2020;35:219-21.

4. Hou S. Pregnancy in dialysis patients: where do we go from here? Semin Dial. 2003;16:376-8.

5. Hladunewich M, Schatell D. Intensive dialysis and pregnancy. Hemodial Int. 2016;20:339-48.

6. Eroğlu D, Lembet A, Ozdemir FN, Ergin T, Kazanci F, Kuşcu E, et al. Pregnancy during hemodialysis: perinatal outcome in our cases. Transplant Proc. 2004;36:53-5.

7. Piccoli GB, Conijn A, Consiglio V, Vasario E, Attini R, Deagostini MC, et al. Pregnancy in dialysis patients: is the evidence strong enough to lead us to change our counseling policy? Clin J Am Soc Nephrol. 2009;5:6271.

\section{FONTES DE FINANCIAMENTO}

Não existiram fontes externas de financiamento para a realização deste artigo.

8. Manisco G, Potì M, Maggiulli G, Di Tullio M, Losappio V, Vernaglione L. Pregnancy in end-stage renal disease patients on dialysis: how to achieve a successful delivery. Clin Kidney J. 2015;8:293-9.

9. Chinnappa V, Ankichetty S, Angle P, Halpern SH. Chronic kidney disease in pregnancy. Int J Obstet Anesth.. 2013;22:223-30.

10. Rashid M, Rashid HM. Chronic renal insuffiency in pregnancy. Saudi Med J. 2003;24:709-14

11. Cabiddu G, Castellino S, Gernone G, Santoro D, Moroni G, Giannattasio $M$, et al. Best practices on pregnancy on dialysis: The Italian Study Group on Kidney and Pregnancy. J Nephrol. 2015;28:279-88.

12. Piccoli GB, Minelli F, Versino E, Cabiddu G, Attini R, Vigotti FN, et al. Pregnancy in dialysis patients in the new millennium: a systematic review and meta-regression analysis correlating dialysis schedules and pregnancy outcomes. Nephrol Dial Transplant. 2016;31:1915-34.

13. Zanlorenci VP, Ribeiro RG, Kahhale S, Zugaib M. Gestação na paciente dialítica. FEMINA. 2009;37:349-55.

14. Bramham K. Dialysis and pregnancy: no longer the impossible. Nephrol Dial Transplant. 2016;31:1763-5.

15. Sulaiman K, Vuppali M, Abreo K. Patient outcome in pregnancy requiring dialysis: a case series. The Open Urol Nephrol J. 2014;7:52-5.

\section{Photosensitive Self-Induced Seizures Since Childhood}

